\title{
Red cell distribution width to lymphocyte ratio as a new biomarker in predicting prognosis in chronic liver disease patients with hepatocellular carcinoma
}

\author{
Yakup Ülger*; Anıl Delik \\ Faculty of Medicine, Department of Gastroenterology, Çukurova University, Adana 01330, Turkey.
}

*Corresponding Author: Yakup Ülger

Çukurova University, Faculty of Medicine, Department of Gastroenterology, Adana, Turkey.

Tel: 0322-338-60-60; Email: yakup73us@yahoo.com

Received: Sep 08, 2021

Accepted: Oct 06, 2021

Published: Oct 11, 2021

Archived: www.jjgastro.com

Copyright: (C) Ülger y (2021).

Keywords: chronic liver disease; HCC; novel predictor; red cell distribution width-to-lymphocyte ratio; prognosis.

Abbreviations: RLR: Red Cell Distribution Width to lymphocyte ratio; NLR: neutrophil-to-lymphocyte ratio; PLR: plateletto-lymphocyte ratio; NMR: Neutrophil-to-Monocytes Ratio; RDW: Red Cell Distribution Width; MPV: Mean Platelet Volüme; OR: Odds Ratio; $\mathrm{Cl}$ : Confidence Interval.

\section{Abstract}

Background: Hepatocellular Carcinoma (HCC), hepatitis B, hepatitis $\mathrm{C}$, chronic alcoholism, non-alcoholic fatty liver disease, copper and iron deposition etiologies, together with various genetic factors, constitute a rather complex cancer group that includes chronic inflammation, chronic systemic inflammation triggered by oxidative stress. Therefore, an increase in the incidence and prevalence of liver cancer is expected in the future. Discovery of new biomarkers in HCC diagnosis, prognosis, prediction of treatment response and treatment follow-up is very important. The aim of our study was to retrospectively evaluate the clinical significance of the Red blood cell Distribution Width (RDW) to lymphocyte ratio $(R L R)$ in patients with chronic liver disease with HCC.

Materials and methods: Our study, which was designed retrospectively, included 200 patients who were followed up with the diagnosis of HCC. Liver function tests, hematological parameters, biochemical tests were analyzed in Çukurova University Balcalı hospital. Child Pugh Score (CTP) and RLR of the patients were calculated with the necessary equations. HCC patients were analyzed in two groups as alfa feto protein (AFP) negative $(<20 \mathrm{ng} / \mathrm{ml})$ and AFP positive ( $>20 \mathrm{ng} / \mathrm{ml}$ ). All the obtained results were analyzed in SPSS program.

Results: A total of 200 patients with HCC were included in this study. Half of the $200 \mathrm{HCC}$ patients included in the study were found to be AFP negative, and the gender distribution in this group was found to be $24.2 \%$ for women and $75.8 \%$ for men. A significant increase in the rate of RLR was detected in the AFP positive group (AFP negative $13.54 \pm 9.67$, AFP positive $20.11 \pm 27.38$, and $p$ value 0.03). According to Child Pugh score, RLR rates were found to be $11.72 \pm 8.82$ in CTP A, $15.96 \pm 8.78$ in CTP B, $27.47 \pm 35.6$ in CTP C, $p$ value $<0.001$. In multivariate logistic regression analysis according to AFP group distribution, the OR value of RLR was determined as $0.95,95 \% \mathrm{Cl}(0.90-1.00)$ and $\mathrm{p}$ value was 0.02 .

Conclusion: We think that RLR can be used as a new biomarker associated with poor prognosis in AFP positive HCC patients. 
Citation: Ülger $Y$, Delik A. Red cell distribution width to lymphocyte ratio as a new biomarker in predicting prognosis in chronic liver disease patients with hepatocellular carcinoma. Japanese J Gastroenterol Res. 2021; 1(5): 1024.

\section{Introduction}

Hepatocellular carcinoma (HCC) is one of the most common and aggressive malignancies of the liver [1]. It is the 4th leading cause of cancer-related death in the worldwide [2]. Chronic hepatitises (such as hepatitis B virus (HBV), Hepatitis C virus (HCV), chronic alcohol consumption, non-alcoholic steatohepatitis are the main risk factors for $\mathrm{HCC}[3]$.

Various classifications are used to predict the prognosis and evaluate liver functional reserve in HCC patients such as ChildPugh (CTP) score, model for end-stage liver disease (MELD) score, Barcellona clinic liver cancer (BCLC) score [4]. HCC often develops in the background of chronic liver disease. liver biopsy still remains the gold standard in the diagnosis of cirrhosis, despite its negative aspects such as being an invasive method, risk of complications, sampling errors, and different evaluations by different pathologists $[5,6]$. Recently, laboratory parameters such as neutrophil-to-lymphocyte ratio (NLR), platelet-tolymphocyte ratio (PLR), neutrophil-to-monocytes ratio (NMR) have been used to evaluate liver function, risk of liver failure after hepatectomy, and survival in HCC patients [7-11]. The goal of this study is to evaluate the role of RLR level in clinical practice as an important biomarker in predicting prognosis in HCC patients.

\section{Materials and methods}

\section{Study population}

The medical records of the patients who were followed up with the diagnosis of HCC in our department between January 2010 and June 2020 were retrospectively reviewed. Exclusion criteria were: mixed hepatocellular cholangiocarcinoma; missing information about its calculation; and co-existing hematological disease. Our study was in accordance with the Declaration of Helsinki [12] and was approved by the Çukurova Medical Faculty Balcalı Hospital Ethics Committee. Written informed consent was obtained from the patients. The HCC diagnostic criteria were based on the guidelines recommended by the European Association for the Study of the Liver (EASL) [13]. We diagnosed HCC when a patient had one or more risk factors: examination using $\alpha$-fetoprotein (AFP) and spiral computed tomography (CT), dynamic MRI with contrast; or AFP and at least two findings following $\mathrm{CT}$, MRI. A positive HCC finding using dynamic CT or MRI is indicative of venous clearance after arterial increase in the delayed portal/venous phase. In addition, we made a histopathological diagnosis in cases that did not meet all clinical noninvasive diagnostic criteria for HCC.

\section{Statistical analysis}

We used SPSS version 18.0 for data entry and analysis. Normally distributed continuous variables (Kolmogorov-Smirnov test, $\mathrm{P}>.05$ ) were expressed as mean value \pm standard deviation (SD). Or the median (interquartile range) was used. Comparisons between groups were made using the $t$, Wilcoxon or $\chi 2$ test, as appropriate. Data divided into two groups showing parametric distribution were evaluated with the Anova test, and the data with non-parametric distribution analyzed in three groups were evaluated with the Kruskal Wallis test. $P$ value $<.05$ was considered statistically significant.

\section{Results}

A total of 200 patients were included in the study. $77.9 \%$ of the patients were male and $22.1 \%$ were female. The mean age of HCC patients was $64.79 \pm 11.41$ years. The patients were evaluated in two groups as AFP negative $(50 \%)$ and AFP positive (50\%). AST level $91.54 \pm 216$ in AFP negative group, 266.13 $\pm 1301 \mathrm{p}$ value 0.001 in AFP positive group, GGT level $109 \pm$ 150, $140 \pm 144$, p value 0.006, ALP level $167 \pm 298,196 \pm 173$, $p$ value 0.001, CRP level $4.42 \pm 6.71,9.74 \pm 16.54$, $p$ value 0.01 , ferritin level $205 \pm 498,397 \pm 766$, p value 0.01, RLR level 13.54 $\pm 54,20.11 \pm 27.38$, $p$ value 0.03 . Other demographic results are shown in (Table 1 ).

Table 1: Demographic data by alpha feto protein distribution in hepatocellular carcinoma patients.

\begin{tabular}{|c|c|c|c|}
\hline Variable & AFP negative $(n=100)$ & AFP positive $(n=100)$ & $P$ value \\
\hline Gender $(n, \%)$ & & & 0.18 \\
\hline Female & $23(24.2)$ & $18(18)$ & \\
\hline Male & $72(75.8)$ & $82(82)$ & \\
\hline Age, (yr) & $64.93 \pm 10.43$ & $64.57 \pm 12.56$ & 0.82 \\
\hline $\mathrm{HGB}, \mathrm{gr} / \mathrm{dl}$ & $12.62 \pm 2.47$ & $12.65 \pm 2.53$ & 0.93 \\
\hline Glucose, mg/dl & $119 \pm 46$ & $129 \pm 73$ & 0.85 \\
\hline Creatinin, mg/dl & $1.12 \pm 0.95$ & $2.06 \pm 8.05$ & 0.28 \\
\hline ALT, U/L & $62.62 \pm 83$ & $124.85 \pm 606$ & 0.06 \\
\hline AST, U/L & $91.54 \pm 216$ & $266.13 \pm 1301$ & 0.001 \\
\hline GGT, U/L & $109 \pm 150$ & $140 \pm 144$ & 0.006 \\
\hline ALB, $g / L$ & $3.24 \pm 0.7$ & $3.29 \pm 4.2$ & 0.90 \\
\hline$A L P, U / L$ & $167 \pm 298$ & $196 \pm 173$ & 0.001 \\
\hline T.BíL, mg/dl & $3.94 \pm 9.6$ & $3.62 \pm 5.4$ & 0.77 \\
\hline D.BìL, mg/dl & $1.54 \pm 3.4$ & $3 \pm 8.6$ & 0.14 \\
\hline Calsium, mg/dl & $9.20 \pm 0.9$ & $9.48 \pm 1.3$ & 0.16 \\
\hline PT & $14.84 \pm 4.18$ & $15.69 \pm 7.32$ & 0.32 \\
\hline INR & $1.26 \pm 0.24$ & $2.79 \pm 13.8$ & 0.29 \\
\hline CRP, mg/dl & $4.42 \pm 6.71$ & $9.74 \pm 16.54$ & 0.01 \\
\hline Ferritin, ng/ml & $205 \pm 498$ & $397 \pm 766$ & 0.01 \\
\hline Child pugh (n,\%) & & & 0.41 \\
\hline Child A & $28(43.8)$ & $29(34.9)$ & \\
\hline Child B & $19(29.7)$ & $24(28.9)$ & \\
\hline Child C & $17(26.6)$ & $30(36.1)$ & \\
\hline RLR & $13.54 \pm 9.67$ & $20.11 \pm 27.38$ & 0.03 \\
\hline
\end{tabular}

HGB: Hemoglobine; ALT: Alanine Aminotransferase; AST: Aspartate Aminotransferase; GGT: Gamma-Glutamyl Transferase; ALB: Albumine; ALP: Alkaline phosphatase; T.BIL: Total Bilirubin; D.BIL: Direct Bilirubin; PT: Prothrombin Time; INR: International Normalized Ratio; CRP: Creactive protein; RLR: Red cell distribution width to lymphocyte ratio. 
Distribution of HCC patients by child pugh score, RLR level $11.72 \pm 8.82$ in Child A group, $15.96 \pm 8.78$ in Child B group, $27.47 \pm 35.6$ in Child C group, p value $<0.001$, CRP level $5.15 \pm$ 14 in Child A group, 6.14 \pm in Child B group 7.88, $12.90 \pm 16.99$ in Child C group, $p$ value $<0.001$, ferritin level $183 \pm 369$ in Child $A$ group, $144 \pm 140$ in Child B group, $652 \pm 1000$ in Child C group, $p$ value 0.001 . All demographic data according to Child Pugh score are given in (Table 2 ).

According to logistic regression analysis according to AFP distribution in HCC patients, RLR level OR $0.9595 \% \mathrm{Cl}$ (0.9-1.00), p value 0.02 , CRP level OR 0.93, $95 \% \mathrm{Cl}(0.87-1.00)$, p value 0.02 , ALP level OR 1.00, $95 \% \mathrm{Cl}(0.99-1.00)$, p value was determined as 0.07 . All of the logistic regression analysis results are given in (Table 3).

Table 2: Distribution of data by Child Pugh score in hepatocellular carcinoma patients.

\begin{tabular}{|l|c|c|c|c|}
\hline \multicolumn{1}{|c|}{ Variable } & Child A & Child B & Child C & P value \\
\hline RLR & $11.72 \pm 8.82$ & $15.96 \pm 8.78$ & $27.47 \pm 35.6$ & $<0.001$ \\
\hline ALT, U/L & $40 \pm 29$ & $193 \pm 922$ & $94 \pm 140$ & $<0.001$ \\
\hline AST, U/L & $51 \pm 40$ & $392 \pm 196$ & $222 \pm 332$ & $<0.001$ \\
\hline GGT, U/L & $103 \pm 104$ & $109 \pm 108$ & $181 \pm 204$ & 0.09 \\
\hline ALP, U/L & $117 \pm 79$ & $163 \pm 115$ & $340 \pm 433$ & $<0.001$ \\
\hline CRP, mg/dl & $5.15 \pm 14$ & $6.14 \pm 7.88$ & $12.90 \pm 16.99$ & $<0.001$ \\
\hline Ferritin, ng/ml & $183 \pm 369$ & $144 \pm 140$ & $652 \pm 1000$ & $<0.001$ \\
\hline ALB, g/L & $4.18 \pm 5.47$ & $2.89 \pm 0.60$ & $2.38 \pm 0.59$ & $<0.001$ \\
\hline
\end{tabular}

ALT: Alanine Aminotransferase; AST: Aspartate Aminotransferase; GGT: Gamma-Glutamyl Transferase ALB: Albumine; ALP: Alkaline Phosphatase; CRP: C-Reactive Protein; RLR: Red Cell Distribution Width To Lymphocyte Ratio.

Table 3: Logistic regression analysis according to AFP distribution in hepatocellular carcinoma patients.

\begin{tabular}{|l|c|c|c|c|}
\hline \multirow{2}{*}{ Variable } & O.R & \multicolumn{2}{|c|}{$95 \% \mathrm{Cl}$} & P value \\
\hline Age, (yr) & 1.03 & 0.98 & 1.07 & 0.13 \\
\hline ALT, U/L & 1.00 & 0.99 & 1.00 & 0.89 \\
\hline AST, U/L & 1.00 & 0.99 & 1.00 & 0.86 \\
\hline GGT, U/L & 0.99 & 0.99 & 1.00 & 0.18 \\
\hline ALP, U/L & 1.00 & 0.99 & 1.00 & 0.07 \\
\hline CRP, mg/dl & 0.93 & 0.87 & 1.00 & 0.02 \\
\hline Ferritin, & 1.00 & 0.99 & 1.00 & 0.74 \\
ng/ml & 0.95 & 0.90 & 1.00 & 0.02 \\
\hline RLR & & & & \\
\hline
\end{tabular}

ALT: Alanine Aminotransferase; AST: Aspartate Aminotransferase; GGT: Gamma-Glutamyl Transferase; ALP: Alkaline Phosphatase; CRP: C-Reactive Protein; RLR: Red Cell Distribution Width To Lymphocyte Ratio.

\section{Discussion}

RDW is a noninvasive, routinely measured hematological parameter that reflects anisocytosis. Recently, it has been reported that RDW may be a prognostic biomarker in predicting the clinical course of various diseases such as cardiovascular diseases, sepsis, liver cirrhosis, autoimmune liver disease, cancers, leukemia, renal dysfunction and respiratory diseases [5,14-16]. $S$ Jing, et al found the RDW level to be an optimal prognostic threshold of $14.15 \%$ in determining survival in HCC patients, and they found RDW level below this value to be associated with low mortality $(p:<0.001)[17]$. Some researchers have reported that the RLR (RDW to lymphocyte ratio) index is a good biomarker in predicting the severity of hepatic fibrosis and cirrhosis in NAFLD patients $[18,19]$. They showed that NAFLD patients had simple adiposity and higher RDW values compared to healthy individuals. It has been reported that increased RDW levels are positively correlated with severe hepatic fibrosis [20]. Wang et al reported that RLR and MPV (platelet volume) were associated with the severity of fibrosis in chronic hepatitis $C$ patients. It is suggested that this will reduce the need for liver biopsy [21].

Zhang et al and Gao et al found that high RLR levels were associated with an increase in HBV-related cirrhosis and hepatitis b viral load [5,22]. On the contrary, Karagöz et al. found no significant relationship between these variables in patients undergoing liver transplantation [23].

In our study, in which the RLR index in HCC was evaluated for the first time in the Turkish population, it was shown that RLR is a biomarker associated with poor prognosis when the AFP negative HCC and AFP positive HCC groups are compared. (p:0.03). The limitations of this study were that it was small sample, single-center, and retrospective. RLR can be used as a biomarker to predict chronic liver disease severity and mortality in HCC patients.

\section{Conclusion}

We evaluate that RLR in AFP positive HCC is a simple, inexpensive and non-invasive new hematological biomarker useful in clinical practice in predicting prognosis.

Conflict of interest: The authors declare no conflict of interests

Author contributions: Conseption and design: YÜ, AD Collection and assembly of data: YU, AD Data analysis: YÜ manuscript writing: $A D$

Competing interests: All authors declare that there is no conflict of interest.

\section{References}

1. Llovet, JM, Kelley RK, Villanueva, A. et al. Hepatocellular carcinoma. Nat Rev Dis Primers. 2021; 7: 6.

2. Wu B, Hu X, Jin H, Zhou L, Zhang $D$, et al. Albumin-bilirubin and platelet-albumin-bilirubin grades for hepatitis B-associated hepatocellular carcinoma in Child-Pugh A patients treated with radical surgery: $A$ retrospective observational study. Medicine. 2019; 98.

3. Yen YH, Cheng YF, Wang JH, Lin CC, Wang CC. Characteristics and etiologies of hepatocellular carcinoma in patients without cirrhosis: When East meets West. Plos one. 2021; 16: e0244939.

4. Ma W, Zhang P, Qi J, Gu L, Zang M, et al. Prognostic value of platelet to lymphocyte ratio in hepatocellular carcinoma: a me- 
ta-analysis. Scientific reports. 2016; 6: 1-6.

5. Zhang X, Wang D, Chen Z, Guo N, Wang W, et al. Red cell distribution width-to-lymphocyte ratio: A novel predictor for HBVrelated liver cirrhosis. Medicine. 2020; 99.

6. Friedman LS. Controversies in liver biopsy: Who, where, when, how, why? Curr Gastroenterol Rep. 2004; 6: 30-6.

7. Ho SY, Liu PH, Hsu CY, Ko CC, Huang YH, et al. Tumor burden score as a new prognostic marker for patients with hepatocellular carcinoma undergoing transarterial chemoembolization. Journal of Gastroenterology and Hepatology. 2021.

8. Goyal H, Hu ZD. Prognostic value of red blood cell distribution width in hepatocellular carcinoma. Annals of translational medicine. 2017; 5.

9. Hu J, Wang N, Yang Y, Ma L, Han R, et al. Diagnostic value of alpha-fetoprotein combined with neutrophil-to-lymphocyte ratio for hepatocellular carcinoma. BMC gastroenterology. 2018, 18: $1-7$

10. Li H, Wang L, Chen L, Zhao H, Cai J, et al. Prognostic value of albumin-to-alkaline phosphatase ratio in hepatocellular carcinoma patients treated with liver transplantation. Journal of Cancer. 2020; 11: 2171.

11. Hu Z, Chen H, Chen S, Huang Z, Qin S, et al. The value of neutrophil to lymphocyte ratio and gamma-glutamyl transpeptidase to platelet ratio in patients with hepatocellular carcinoma. Medicine. 2019; 98

12. World Medical A. World Medical Association Declaration of Helsinki: ethical principles for medical research involving human subjects. JAMA. 2013; 310: 2191-4.

13. Bruix J, Sherman M, Llovet JM et al. Clinical management of hepatocellular carcinoma. Conclusions of the Barcelona-2000 EASL conference. European Association for the Study of the Liver. Hepatol. 2001; 35: 421- 430.

14. Jo YH, Kim K, Lee JH, et al. Red cell distribution width is a prognostic factor in severe sepsis and septic shock. Am J Emerg Med. 2013; 31: 545-8.
15. Zhao, T, Cui, L, Li, A. The significance of RDW in patients with hepatocellular carcinoma after radical resection. Cancer Biomarkers. 2016; 16: 507-512.

16. Montagnana M, Danese E. Red cell distribution width and cancer. Ann Transl Med 2016; 4: 399.

17. Jing JS, Fu XL, Zhao W, Kong LB. Red Cell Distribution Width as a Prognostic Factor in Patients with Hepatocellular Carcinoma. Clinical laboratory. 2020; 66.

18. Cengiz M, Ozenirler S. Comparative diagnostic accuracy of red cell distribution width-to-platelet ratio versus noninvasive fibrosis scores for the diagnosis of liver fibrosis in biopsy-proven nonalcoholic fatty liver disease. Eur J Gastroenterol Hepatol. 2015; 27: 1293-1299.

19. Taefi A, Huang CC, Kolli K, Ebrahimi S, Patel M. Red cell distribution width to platelet ratio, a useful indicator of liver fibrosis in chronic hepatitis patients. Hepatol Int. 2015; 9: 454-46

20. Zhou WJ, Yang J, Zhang G, Hu ZQ, Jiang YM, Yu F. Association between red cell distribution width-to-platelet ratio and hepatic fibrosis in nonalcoholic fatty liver disease: A cross-sectional study. Medicine. 2019; 98.

21. Wang Q. et al. The Severity of Liver Fibrosis Influences the Prognostic Value of Inflammation-Based Scores in Hepatitis B-Associated Hepatocellular Carcinoma. Annals of surgical oncology. 2015; 22: 1125-S1132.

22. Gao P, Xiao P, Yang YL, Chen QF, Mao XR, et al. Effects and clinical significance of virus load on red blood cell parameters in different stage of hepatitis B. Beijing da xue xue bao. Yi xue ban= Journal of Peking University. Health Sciences. 2014; 46: 941-944.

23. Karagoz E, Ulcay A. Tanoglu, A. Kara, M, Turhan, V, Erdem H. \& Gorenek, L. Clinical usefulness of mean platelet volume and red blood cell distribution width to platelet ratio for predicting the severity of hepatic fibrosis in chronic hepatitis B virus patients. European journal of gastroenterology \& hepatology. 2014; 26: 1320-1324. 